Meta

Journal des traducteurs

Translators' Journal

\title{
La traduction de la littérature tunisienne en langues étrangères (Beït Al-Hikma: 17 et 18 avril 1998)
}

\section{Mohamed Salah Ben Amor}

Volume 45, numéro 3, septembre 2000

La traduction dans le monde arabe

URI : https://id.erudit.org/iderudit/001884ar

DOI : https://doi.org/10.7202/001884ar

Aller au sommaire du numéro

Éditeur(s)

Les Presses de l'Université de Montréal

ISSN

0026-0452 (imprimé)

1492-1421 (numérique)

Découvrir la revue

Citer cet article

Ben Amor, M. S. (2000). La traduction de la littérature tunisienne en langues étrangères (Beït Al-Hikma: 17 et 18 avril 1998). Meta, 45(3), 565-567.

https://doi.org/10.7202/001884ar d'utilisation que vous pouvez consulter en ligne. 


\section{La traduction de la littérature tunisienne en langues étrangères (Beït Al-Hikma: 17 et 18 avril 1998)}

Les 17 et 18 avril 1998, s'est tenu à Beït Al-Hikma à Carthage (Tunisie) un colloque sur le thème: «La traduction de la littérature tunisienne en langues étrangères» organisé par l'Académie des sciences humaines, des lettres et des arts avec la collaboration d'un groupe de chercheurs appartenant à 
l'Université de Tunis I. Ce colloque a été dédié au professeur Abdelkader Méhiri, l'un des fondateurs de l'Université tunisienne.

La séance inaugurale a débuté par trois allocutions présentées par M. Abdelwahab Bouhdiba, président de l'Académie, M. Abderraouf Mahbouli, président de l'Université de Tunis I et M. Mohamed Salah Ben Amor, coordinateur du groupe. Les trois intervenants ont insisté sur l'intérêt majeur que les hommes de lettres tunisiens doivent accorder à la traduction de la littérature nationale en langues étrangères; et ce, pour faire face aux menaces de la mondialisation qui présente un danger réel pour l'identité culturelle du pays. Ils ont mis en exergue les qualités scientifiques du professeur A. Méhiri qui est, de l'avis de tous les intervenants, l'un des piliers de l'Université tunisienne.

$$
\star * *
$$

Au cours de la première séance scientifique, présidée par M. Mahmoud Tarchouna, M. Mohamed Yaalaoui a présenté une étude comparative portant sur deux traductions françaises d'Al-Muqaddima d'Ibn Khaldûn : celles de De Slane, un classique, et de Vincent Monteil, un moderniste. Les différences les plus notables dégagées par l'intervenant se situent au niveau des termes techniques utilisés par les deux traducteurs, traditionnels chez le premier et relevant des sciences sociales et économiques modernes chez le second.

La deuxième intervention présentée par M. Mohamed El Kadhi a été consacrée à la traduction française de «la genèse de l'oubli» du narrateur tunisien Mahmoud Messadi (né en 1911) élaborée par Taoufiq Baccar. Cette traduction a eu le mérite, selon l'intervenant, de réaliser un équilibre harmonieux entre la fidélité au texte original et la non soumission à son style arabe classique.

Une troisième intervention, celle de Mme Fawzia Saffar Zaouq, propose une approche critique de la traduction française du récit «Périple à travers les bars méditerranéens " d'Ali Douagi (1909-1949). Cette traduction réalisée par un cinéaste tunisien $\mathrm{M}$. Tahar Chériaa, comporte d'après $\mathrm{M}^{\text {me }}$ Zaouq, plusieurs défauts tels que les faux-sens, les contresens, les pléonasmes et le calque des structures, en plus des fautes flagrantes de grammaire et d'orthographe; d'où la nécessité d'une autre traduction qui doit être faite par un traducteur de métier maîtrisant parfaitement les deux langues.

M. Jean Fontaine s'est penché, de son côté, sur la traduction française du roman «Les dattes dans leurs régimes» de Béchir Khraïef (19171983) élaborée par Hédi Jebnoun et Assia Djebar. Il y relève l'omission de pas moins de 1037 lignes représentant $10 \%$ du roman. Les passages «censurés» portent sur deux sujets: les relations amoureuses et la politique. Ce qui amène l'intervenant à conclure que «ces omissions laissent penser à une manipulation, fut-elle inconsciente».

Enfin la traduction anglaise du fameux ouvrage «Aqwam al Masalak» (Les voies les plus sûres) de Kheireddine Ettounsi (1889), réalisée par Leon Carl Brown, a été examinée minutieusement par Hefedh Brini qui confirme le respect du texte original malgré l'incohérence et l'imprécision flagrantes au niveau terminologique.

$$
\star * *
$$

La deuxième séance scientifique présidée par M. Mohamed Salah Ben Amor a comporté quatre interventions. Dans la première, $\mathrm{M}^{\mathrm{lle}}$ Chédia Trabelsi a tenté une comparaison exhaustive entre deux traductions françaises des "Pensées » de Tahar Haddad (1901-1935), élaborées par deux Tunisiens: Noureddine Sraïeb et Hédi Balegh. La conclusion essentielle à laquelle a abouti ce travail comparatif est la fidélité des deux traductions au texte original, avec toutefois, une réussite plus nette de $\mathrm{H}$. Balegh dans le respect des caractéristiques du style français.

Le deuxième intervenant, M. Rafiq Ben Ouennes, a été le seul participant à émettre un avis uniquement favorable sur la traduction qu'il venait d'examiner. Cette traduction, en l'occurrence celle de quelques poèmes d'Abu-Al-Qasim Echabbi (1909-1934) par Ameur Ghédira, est la seule, selon $\mathrm{M}$. Ben Ouennes, à avoir atteint un haut degré de perfection.

M. Taïeb Achach, quant à lui, a brossé un tableau général des travaux de traduction vers les langues étrangères se rapportant à la littérature tunisienne. Ces travaux se caractérisent, surtout, par la précarité des critères adoptés dans le choix des textes ainsi que par leur faiblesse due à la non maîtrise de la technique de traduction proprement dite et des deux langues: source et but.

M. Féthi Gasmi a examiné, de son côté, les traductions françaises de deux épîtres arabes écrites au XIX ${ }^{e}$ siècle par Mohamed Senoussi (18501900). Ces épîtres dont les textes originaux n'ont pu être trouvés ont pour titres: «Lettre sur la Majba» et «l'épanouissement de la fleur». Et étant dans l'impossibilité de procéder à une étude comparative, l'intervenant s'est contenté d'analyser le contenu de ces deux textes, mettant en exergue les idées novatrices et nationalistes de cet éminent réformateur tunisien.

$$
* * *
$$

La troisième séance scientifique, présidée par $\mathrm{M}$. Taïeb Baccouche, n'a comporté qu'une seule intervention traitant d'une traduction vers le français. En effet, elle a offert l'occasion à quatre autres chercheurs d'étudier des traductions d'ouvrages 
littéraires tunisiens vers l'italien, le russe, l'espagnol et l'anglais.

La traduction française en question et celle des «Mémoires d'Echabbi» par Mongi Chemli et Mohamed Ben Ismaïl. Cette traduction a été l'objet d'une étude présentée par M. Ahmed Remadi qui y a décelé, malgré quelques inconhérences sans gravité, un grand effort de transmission des idées de Chebbi dans un style purement français. $\mathrm{M}^{\mathrm{me}}$ Samia Weslati Fattoum a étudié, pour sa part, quatre nouvelles du narrateur tunisien Rached Hamzaoui (né en 1932), traduites en italien par Linda Bettini. Après un examen critique de cette traduction, elle conclut que le travail de Mme Bettini doit être repris de fond en comble en regard des dizaines de faux-sens dûs à une faible connaissance de l'arabe classique et de l'arabe dialectal tunisien.

M. Mohsen Tounsia a donné un aperçu général des œuvres littéraires tunisiennes traduites jusqu'à ce jour en russe. Le bilan est, selon lui, très négatif. Et ce en raison du mauvais choix des textes traduits. Certains sont médiocres. D'autres ont été écrits par des étrangers vivant en Tunisie ou par des Tunisiens liés au colonialisme, sans compter les défauts de traduction purement techniques, d'où la nécessité de mettre sur pied un comité de traducteurs tunisiens maîtrisant le russe et l'arabe et d'établir un programme national comportant un choix rigoureux des œuvres tunisiennes à traduire vers la langue de Tolstoï.

La langue espagnole a été présente dans ce colloque grâce à l'intervention de $\mathrm{M}$. Mohamed Ali Ben M'rad qui a analysé la traduction vers cette langue du roman «Barg Ellil» de Béchir Khraïef, réalisée par Ana Ramos.

Malgré le nombre élevé de fautes relevées dans le texte espagnol, l'intervenant n'a pas manqué de louer l'effort déployé par la traductrice pour reproduire les sens du texte original, en dépit de sa méconnaissance du dialecte tunisien utilisé fréquemment par l'auteur dans les dialogues.

La dernière intervention a été présentée par M. Fathi Dali qui a proposé une lecture de la traduction anglaise faite par l'américain William Granara du recueil de nouvelles intitulé «Il m'a fait veiller tant de nuits» d'Ali Douagi.

Cette traduction est, selon M. Dali, une véritable déformation du texte original, car elle a fait abstraction de l'essence même de ce texte qui réside dans son ton humoristique et ironique.

Ainsi pouvons-nous constater que la plupart des traductions étudiées, au cours de ce colloque, ont fait l'objet de critiques sévères à cause de leur nonconformité avec les textes originaux ou tout sim- plement de leur faiblesse due à l'improvisation ou à la rapidité d'exécution ou à la connaissance superficielle de la lange arabe.

Cette constatation non réjouissante a amené les participants, au cours des débats, à conclure que les œuvres littéraires tunisiennes de valeur ne peuvent, en aucun cas, rester à la merci des traductions individuelles qui atteignent, rarement, la perfection recherchée. Bien entendu, l'acte de traduction est personnel, donc libre, et on ne peut interdire à quiconque de l'accomplir. Mais l'intérêt national impose de réaliser une traduction de qualité des ces œuvres qui constituent un patrimoine bien ancré dans la mémoire collective des tunisiens.

Des traductions de cette classe ne peuvent, cependant, être élaborées que par un groupe multilingue alliant l'expérience et la maturité des anciens à l'ardeur et l'ambition des jeunes. Un tel groupe peut être, facilement, mis sur pied; car la Tunisie compte des dizaines de traducteurs maitrisant un grand nombre de langues. Pour ce faire, il suffit qu'une coopération entre l'Université de Tunis I et l'Académie des sciences, des lettres et des arts soit mise en place.

\section{Mohamed Salah Ben Amor}

\section{RÉFÉRENCES}

Khayr al-Din al-Tunisi (1967): The Surest Path (trad. Leon Carl Brown), Harvard, Harvard Middle Eastern Monographs.

Снавві Авоu al-Qасім (1988): «Journal» (trad. Mongi Chemli et Mohamed Ben Ismail), Carthage, Fondation nationale.

Снeвbi, A. (1959): Choix de poèmes (trad. Ameur Ghédira), Paris, Seghers.

DouAgI, A. (1991): «Sleepless nights» (trad. William Granara), Carthage, Fondation nationale.

— (1979): Périple à travers les bars méditerranéens, Tunis, M.T.E.

Guckin De Slane, M. (1963): Prolégomènes historiques d'Ibn Khaldûn, Paris.

Haddad, T. (1984): Pensées ( $1^{\text {re }}$ trad. Noureddine Sraïeb, Oran; 1996: $2^{\mathrm{e}}$ trad. Hédi Balegh, Tunis).

Hamzaoui, R. (1978): Quatro novelle. Istituto per l'Oriente (trad. Linda Bettini), Rome.

Khraïef, B. (1982): Barg Al-Lil (trad. Ana Romas), Madrid.

LATÈs, J.-C. (1986) : La terre des passions brûlées (trad. Hédi Djebnoun et Assia Djebar), Paris.

Messadi, M. (1993): La génèse de l'oubli (trad. Taoufiq BACCAR), Carthage, Académie tunisienne.

MonteIL, V. M. (1968): Discours sur l'Histoire Universelle, Paris, UNESCO.

SEnoussi, M. (1897) : «L'épanouissement de la fleur », Revue Tunisienne, 10 (juillet 1897).

_ (1896) : «Lettre sur la Mejba», Revue Tunisienne, 9 (janvier 1896), $3^{\mathrm{e}}$ année. 